\title{
Study on Availability of Damping Device Design of A Photoetching Machine
}

\author{
Qiguang Ke \\ Wenzhou Vocational \& Technical College, Wenzhou. 325035 \\ qiguang@163.com
}

Keywords: photoetching machine. device. study.

\begin{abstract}
The basal plate is key component of thin film transistor(TFT), which is mainly used to load bearing processed liquid crystal plate. To decrease the effect of ground shaking, the basal plate is usually fixed on the inner frame supported by the damping device. In this case, in addition to quarantine ground base, the damping device must quickly decrease the vibration caused by horizontal movement reaction force of the basal plate, and compensate the position fluctuation of the bumper caused by change of gravity center when the basal plate moves. With the continuous increase of the size of liquid crystal plate, the basal plate, quality and speed also continuously go up and the reaction force is also stronger and stronger that is reacted onto the inner framework, thus the requirements for the bumper become more and more serious.
\end{abstract}

\section{Background}

At the aspect of compensating the reaction force of the basalt plate movement, the U.S. published a bumper that could use the voice coil motor in the form of flat plate to press control over the inner frame. However, due to little output force of the voice coil motor in the form of flat plate. As the reaction force of the basal plate movement increases, the number of the motor must be added that not only increases cost but also needs to add the number of heat abstraction. But a Japanese bumper adopts the single air cavity to press control force over the inner frame, but to increase horizontal control force, the bumper must enlarge the surface area of the air cavity so as to add the height of the bumper and bring disadvantages. For the change of gravity center when compensating the basal plate moving, both bumpers stated adopt a vertical single direction air cavity to press control and upward control force comes out by restarting the air cavity, and exhausting gas from the air chamber can use the load of gravity to produce downward control.With the increase of substrate quality, as speeding,the damping force compensation control required also faster and faster, there is a certain bottleneck of this program.

\section{Design Content}

A damping device includes a frame body, a vertical piston, a horizontal piston and a plurality of diaphragms, among of which the top of the frame body is linked with the internal frame and the frame body is connected with the foundation at a distance. The vertical piston is disposed in the frame body and passes through the diaphragm and the frame body form a first air cavity and a second air cavity, the horizontal piston is also arranged in the frame body, and form a third air cavity and a fourth air cavity through the film and the frame body. As Fig. 1 shows: 


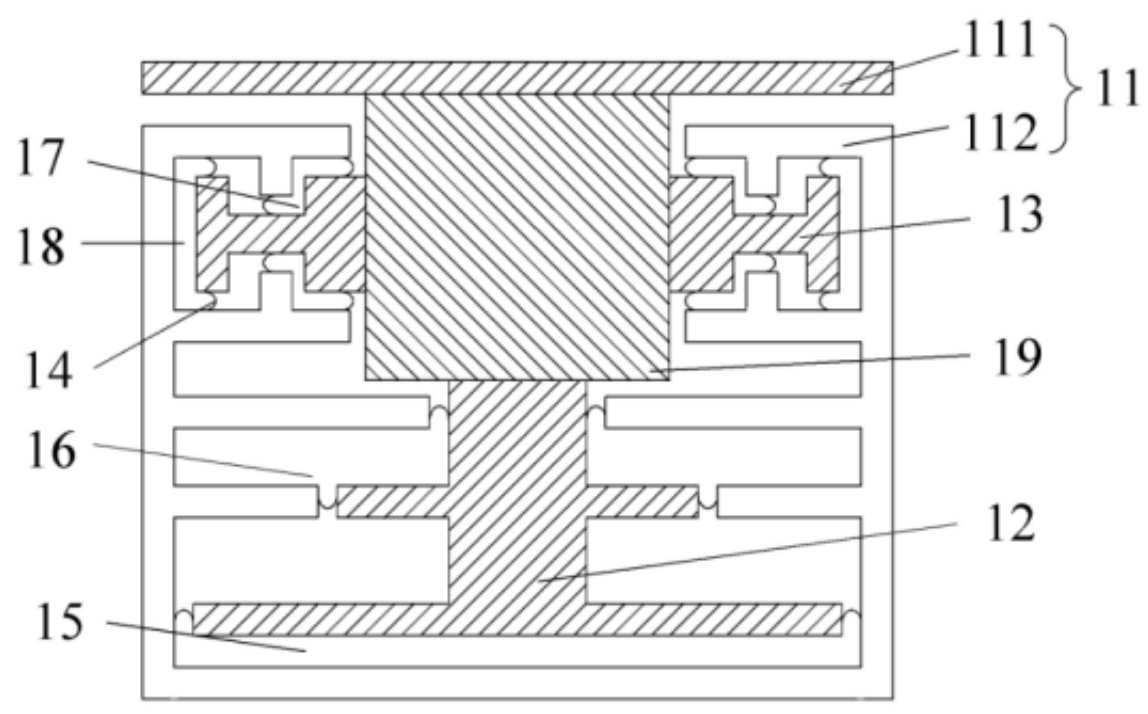

Fig. 1 Damping Device

The effective action area of the piston in the first air cavity is bigger than that of the second air cavity.

The frame includes a top plate and a base.The vertical piston is arranged in the base and connected with the top plate, the horizontal piston is arranged in the top plate and connected with the base, and the vertical piston is horizontally above the piston. As Fig.2 shows:

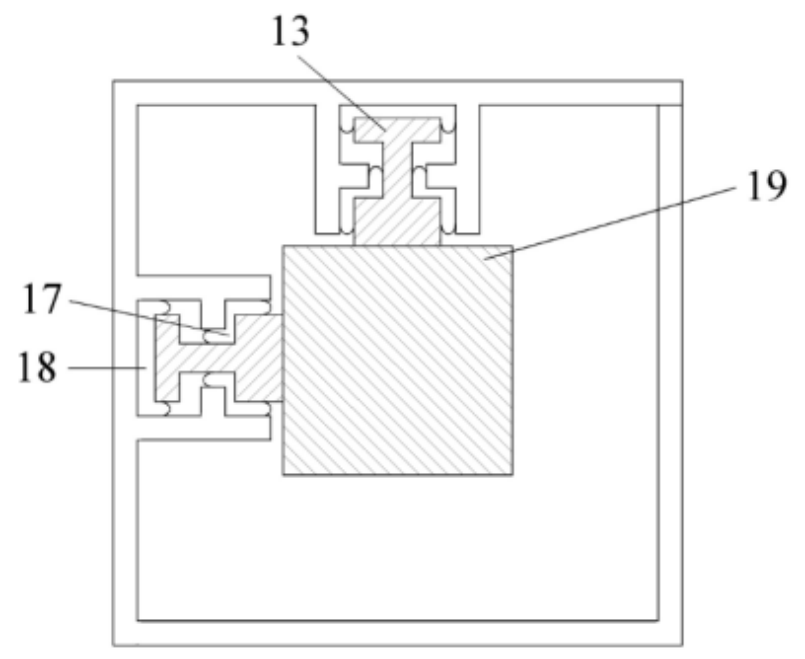

Fig. 2 Piston

The frame body includes the top plate and the base, both the vertical piston and the horizontal piston are set in the base. The vertical piston is connected to the top plate by a connection plate; and the horizontal piston is fixed onto the connection plate. The vertical piston is below the horizontal piston.

The number of the horizontal pistons is four. When there are two horizontal pistons, the two shows to the opposite or adjacent in 90 degrees. When there are four horizontal pistons, the four shows to the opposite in the form of a crossing. The first air cavity and the second air cavity are connected to the external air source through a servo valve and a pressure regulating valve.The third air cavity is connected with the outside air source through a servo valve and a pressure regulating valve.The fourth air cavity is connected with the external air source through a servo valve and a pressure regulating valve. As Fig. 3 shows: 


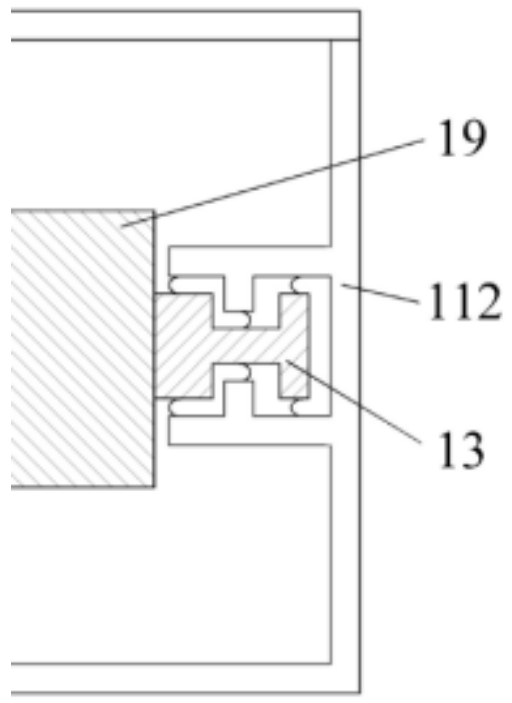

Fig.3 Horizontal Piston

In the horizontal structure, corresponding to the current technological air cavity with the singlelayer structure, the invention adopts the double-layer air cavity structure between the third air cavity and the fourth air cavity, which enlarges the effective surface area. Under the circumstance without adding the height of damping device, the horizontal control force is greatly strengthened. As Fig.4 shows:

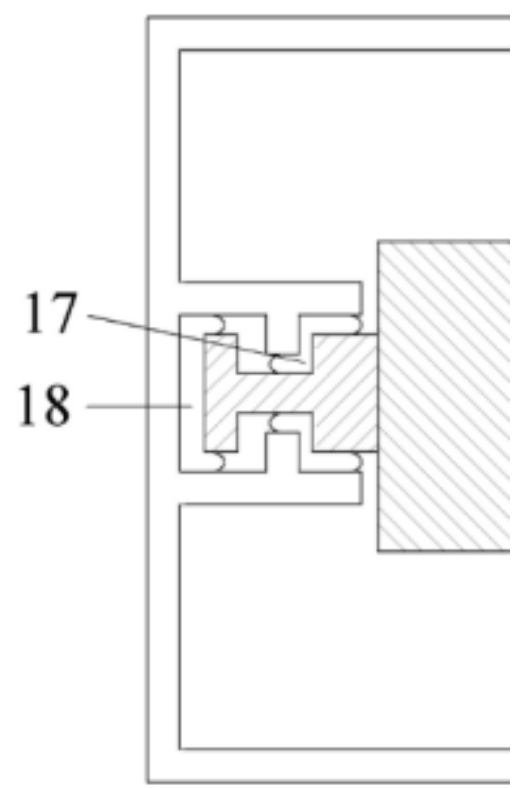

Fig. 4 Horizontal Structure

\section{Implementation Manners}

To make the objectives, the features and advantages of the invention stated above seems simpler, detailed explanations are as follows in combination with additional charts. What is illustrated is the additional chart of the invention is in the form of simplicity and adopts precise proportion, so as to illustrate the objectives of the invention in a convenient and clear way. The present invention provides a shock absorbing device which can increase the horizontal control force, the control cost and the heat output of the shock absorbing device without increasing the height of the shock absorbing device, and can also increase the vertical control force of the vibration damping device .

To apply force two-way force onto Piston 12, in detail, it is for the structure of air cavity. KPA $^{2}$

$\mathrm{K}=----$ 
$\mathrm{V}$,

Stiffness of air suspension, in the formula

K: Adiabatic coefficient;

$\mathrm{P}$ : Absolute pressure in the air cavity;

A: Surface area of air cavity;

V: Volume of air cavity.

Combining the circumstances of the implementation, the vertical stiffness calculation formula for the damping device can be reached:

PV is the absolute pressure in the first air cavity15, Av, is the effective area of the piston in the first air cavity 15, VV1 is the volume of the first air cavity15, and PV2 is the absolute pressure in the second air cavity16.

The damping device can make the load bearing $G$ that is gravity to speed up. Due to the first air cavity 15 is to overcome the load bearing quality of the damping device, the volume of the first air cavity 15 is bigger than that of the second air cavity 16 to make sure the air pressure in the first air cavity 15 can overcome the vertical piston 12 to offer the control force upward.

When the controlled air pressure becomes stronger controlled by the servo valve 10a in Air Pressure 1 existing the third air cavity and the fourth air cavity, the air pressure controlled by the servo valve 10b of the opposite two air cavities becomes weaker, thus the control force is implemented to the right direction. Conversely, the control force is implemented to the left direction.

The invention can not only increase the horizontal control force of the vibration damping device but also increase the control cost and the heat output without increasing the height of the vibration damping device, and can also increase the speed of the five vertical control forces of the vibration damping device.

In support of the load case, the photoetching machine is generally composed of more than three damping devices forming a damping system to support the load. The general damping system consists of three or four damping devices, which can have a variety of forms of five horizontal air cavities. The inventions is not to give more accounts.

\section{Conclusion}

Comparing with the existing technologies on the vertical structure, the invention uses the structure of double air cavities with the first air cavity and the second air cavity, which not only can make the force upward, but also can make the force downward. Thus, the speed of the vertical control force grows up for the damping device. That is to say, the horizontal control force of the damping device is added and the control cost and heating increase under the circumstances without increasing the height of the damping device.

\section{References}

[1]XU Hong-wei. Research on the Innovative Design of Multi-functional Bed with Table[J]. Furniture \& Interior Design, 2016(4): 46—47.

[2]JIANG Man-qun. Research on the Application of Green Design Concept in Children's Product Design[J]. Design, 2017, 30(3): 14-16.

[3]ROBERTA L. Dr. Lee's Decompression[M]. Beijing: China Renmin University Press, 2012.

[4]ZHANG Jie-yu, GAN Jing. Research on Emotional Design of Pressure Relieving Product[J]. Packaging Engineering, 2011, 32(2): 48-51. 